\title{
Three Remarks on Axisymmetric Stationary Horizons
}

\author{
P. Hájiček \\ Institute for Theoretical Physics, University of Berne, Berne, Switzerland
}

Received October 11, 1973

\begin{abstract}
In the first remark the catalogue of axisymmetric stationary horizons is completed by a subset of uncharged translation-symmetric horizons, which was ignored in the previous paper [5]. The subset consists of two one-parameter families: extreme Kerr horizon $(a=m)$ and a more symmetric family. In the second remark the surface area, net angular momentum and net charges of a black hole are computed. It turns out that the four invariant functions $A, B, C, D$ used in [5] to classify the horizons describe, at least formally and up to a constant factor: $A$ the profile of the black hole surface, $B$ the surface density of angular momentum and $C \cos D, C \sin D$ the surface density of electric and magnetic charge. In the third remark a simplified model of a black hole surrounded by a charged matter shell is found to satisfy a sort of generalized "no-hair-conjecture". An example of a non-Kerr-Newman field around a horizon is provided; the magnetic field in it is hoped to have some astrophysical importance.
\end{abstract}

\section{Introduction}

The search for black holes in close binaries (see, e.g. [1]) has shifted the attention from pure vacuum black holes to systems consisting of a hole surrounded with matter disks, rings and shells $[2,3]$.

If there is only vacuum or electrovacuum outside a black hole, then the well-known "no-hair-theorems" [4] tell us what the equilibrium (= stationary) state of the horizon and the field outside it will be: for static electrovacuum it is the Reissner-Nordström family and for stationary vacuum a two-parameter family which, if it contains the Schwarzschild solution, must be that of Kerr. No proof is attempted for stationary electrovacuum, but one conjectures that the Kerr-Newman solution is all what is possible.

A ring of matter surrounding the hole will deform the horizon and influence the field in its neighbourhood. In [5] (the last two papers of [5] will be denoted by I and II, and, e.g. the equation $(x)$ of II by II $(x)$ ) an attempt was undertaken to determine what the equilibrium structure of such horizons and neighbouring fields can be. The method used was based on solving the characteristic initial data constraints for EinsteinMaxwell equations together with that part of Killing equations and their integrability conditions which concerns the inner structure of the 
horizon hypersurface. In such a way all equilibrium horizon configurations can be obtained (including counterexamples to stationary electrovacuum "no-hair-conjecture", if they exist). The axisymmetric stationary families found in II were classified by means of a set of invariants. On the other hand, the question of what external field corresponds to a given set of these invariants is not simple to answer.

In the present paper, we investigate some further interesting properties of these horizons. In Section 2, a small subset of them which was "forgotten" in II is described. With this subset, our catalogue of axisymmetric stationary horizons is completed. It consists of two oneparameter families, one of then being the extreme $\operatorname{Kerr}(a=m)$ while the second, which has more symmetry, has not yet been identified with the horizon of any known solution (such solutions may exist, but if they do they are unknown to the author). There is no net charge, but some electromagnetic field can be present at the horizons of both families. The space-times which contain a horizon of this subset can be very numerous (e.g. for the Kerr horizon, it need not always be the Kerr solution, see II).

In Section 3 we calculate the surface area, net angular momentum and net electric and magnetic charges of the corresponding black hole from our horizon invariants $A, B, \operatorname{Re} \Phi_{1},-i \operatorname{Im} \Phi_{1}$. We arrive at four simple alike integral formulas, which formally allow us to interpret $B, \operatorname{Re} \Phi_{1}$, $-i \operatorname{Im} \Phi_{1}$ (up to some $\pi$-factors) as surface densities of angular momentum, electric and magnetic charge, respectively. Thus, our choice of the invariants, which was dictated by purely mathematical convenience in II, need not be so incoherent and accidental as it looked at the first sight.

In the last section the axisymmetric stationary perturbation analysis of the Schwarzschild field due to Vishveshwara and Price is used to construct a model of a black hole surrounded by a thin matter shell. A formulation of a generalized "no-hair-conjecture" is motivated in such a way and shown to be correct at least in the neighbourhood of Schwarzschild solution. The differential equations for the perturbations between the horizon and the shell can be solved by polynomials for odd gravitational and all electromagnetic modes. We use this to show how the magnetic field of a shell in the neighbourhood of the hole may appear. A proposal that such a field could have an astrophysical significance is considered.

\section{2. $A T$-Horizons with $\Phi_{1}=0$}

In II the differential equations for the axisymmetric horizons with translational longitudinal group (AT-horizons) were divided into three groups. The first group system was shown to allow only Kerr-Newman 
values of $\Gamma, \Omega, \Psi_{2}, \Phi_{1}$ as its solutions. This does not mean, however, that any AT-horizon belongs to the Kerr-Newman family, because the horizon structure (metric and affine connection, see I) is first determined, if one knows the additional quantities $\lambda$ and $\mu$. They must satisfy, together with $\Psi_{3}$ and $\Phi_{2}$, the underdetermined second group system

$$
\left.\begin{array}{r}
\left(M^{+}+\Gamma^{+}+5 \Omega^{+}\right) \Psi_{3}+2 \Phi_{1}^{+}\left(M^{+}+\Gamma^{+}+\Omega^{+}\right) \Phi_{2}-\left(3 \Psi_{2}+4 \Phi_{1}^{+} \Phi_{1}\right) \lambda=0, \\
\left(M^{+}+\Omega^{+}\right) \mu-(M-2 \Gamma+\Omega) \lambda-\Psi_{3}+2 \Phi_{1}^{+} \Phi_{2}=0,
\end{array}\right\}
$$

where $\Gamma, \Omega, \Psi_{2}, \Phi_{1}$ is a solution of the first group. The system (1) was solved under the condition that $\Phi_{1}$ is not zero, i.e. $v<1 / \sqrt{2}$ in the Lemma 13 of II. In such a way, an important subclass of horizons was ignored. A well-known member of this class is the extreme Kerr horizon $(a=m)$. The purpose of the present section is to fill the gap.

The system (1) holds for the values of $\lambda, \mu, \Psi_{3}, \Phi_{2}$ at the Cauchy surface $\alpha=0$, computed in a frame $L, M$, with $M$ tangential to the surface $\alpha=0$ (or else there will appear additional terms in (1); for the meaning of $\alpha, L, M$, see II). All transformations leaving (1) invariant are, therefore, generated by

$$
\begin{aligned}
& \alpha^{\prime}=\alpha-X(\vartheta), \quad L^{\prime}=L, \quad M^{\prime}=M+\xi(\vartheta) L, \\
& \alpha^{\prime}=\eta \cdot \alpha, \quad L^{\prime}=\frac{1}{\eta} L, \quad M^{\prime}=M,
\end{aligned}
$$

where $\eta$ is a real constant and $X(\vartheta), \xi(\vartheta)$ are real functions. They are not independent of each other, because $M^{\prime}$ must be tangential to the surface $\alpha^{\prime}=0$, e.g.

$$
\left(M^{\prime}(\alpha-X(\vartheta))\right)_{\alpha=X}=0,
$$

or, using the relation $L \alpha=1, M \alpha=-\Omega \alpha$, (see I)

$$
\xi=(M+\Omega) X \text {. }
$$

The transformations of $\mu$ and $\lambda$ under (2) [see I (7), I (14)] are

$$
\begin{aligned}
\left.\mu^{\prime}\right|_{\alpha=X} & =\left.\mu\right|_{\alpha=0}+\Psi_{2} X+(M-\Gamma+\Omega) \xi^{+}, \\
\lambda^{\prime} & =\lambda+\left(M^{+}+\Gamma^{+}+\Omega^{+}\right) \xi^{+},
\end{aligned}
$$

under (3) are

$$
\mu^{\prime}=\eta \cdot \mu, \quad \lambda^{\prime}=\eta \cdot \lambda,
$$

where the superscript "+" means complex conjugation. This transformation can be used to simplify the system (1) or to reduce the number of the second group variables (in II, e.g. $\alpha=0$ was chosen to be generated 
by geodesics starting at the pole $\vartheta=0$ orthogonally to the rotation axis. Then, $\left.\mu\right|_{\alpha=0}=-\operatorname{Re} \lambda$ ).

Setting $\Phi_{1}=0$ in (1), we obtain

$$
\begin{aligned}
\left(M^{+}+\Gamma^{+}+5 \Omega^{+}\right) \Psi_{3}-3 \Psi_{2} \lambda & =0 \\
\left(M^{+}+\Omega^{+}\right) \mu-(M-2 \Gamma+\Omega) \lambda-\Psi_{3} & =0
\end{aligned}
$$

or: 1) the electromagnetic variable $\left.\Phi_{2}\right|_{\alpha=0}$ becomes uncoupled from the geometric variables and can be chosen arbitrarily, 2) the system (8) for the geometric quantities is not underdetermined any more because of the gauge freedom. One of its solutions are the extreme Kerr values of $\lambda,\left.\mu\right|_{\alpha=0}$ and $\left.\Psi_{3}\right|_{\alpha=0}$. Are there any others?

Let $v=\frac{a}{\sqrt{a^{2}+m^{2}}}=1 / \sqrt{2}(m=a)$ in the Lemma 13 of II. Then, a canonical frame and the first group quantities are, according to the lemma, given by

$$
\begin{aligned}
L= & \frac{\partial}{\partial \alpha}, M=\frac{1}{\sqrt{2}} \frac{1}{m} \frac{1}{\sqrt{1+\cos ^{2} \zeta}} \\
& \cdot\left(\frac{\partial}{\partial \zeta}+\frac{1+\cos ^{2} \zeta}{2} \frac{i}{\sin \zeta} \frac{\partial}{\partial \varphi}-\alpha \cdot \frac{i \sin \zeta}{1+i \cos \zeta} \frac{\partial}{\partial \alpha}\right) \\
\Gamma= & \frac{1}{\sqrt{2}} \frac{1}{m} \frac{1}{\sqrt{1+\cos ^{2} \zeta}}\left(-2 \frac{\operatorname{ctg} \zeta}{1+\cos ^{2} \zeta}\right), \\
\Omega= & \frac{1}{\sqrt{2}} \frac{1}{m} \frac{1}{\sqrt{1+\cos ^{2} \zeta}}\left(-\frac{i \sin \zeta}{1+i \cos \zeta}\right), \\
\Psi_{0}= & \Psi_{1}=\Phi_{0}=\Phi_{1}=0, \quad \Psi_{2}=-\frac{1}{m^{2}} \frac{1}{(1-i \cos \zeta)^{3}} .
\end{aligned}
$$

Theorem 7. All solutions of (8) corresponding to (9)-(11) define two one-parameter families of horizons (the parameter is $m$ ), viz

(a) The extreme Kerr horizon family, and

(b) A second family characterized by the existence of a canonical frame in which

$$
\lambda=0,\left.\quad \mu\right|_{\alpha=0}=0,\left.\quad \Psi_{3}\right|_{\alpha=0}=0 .
$$

The horizons of this family have an additional symmetry, namely the collineation group generated by $\alpha \frac{\partial}{\partial \alpha}$. 
The quantities $\Phi_{2}$ and $\Psi_{4}$ along a horizon of any of the two families which

1) satisfy the Einstein-Maxwell characteristic initial value constraints together with the metric and affine connection of the horizon,

2) do not break the axial and translation symmetry of the horizon [but they can break the additional collineation symmetry in case (b)] are specified, if one prescribes four real invariant functions of one variable (viz. $\left.\Phi_{2}\right|_{\alpha=0}$ and $\left.\Psi_{4}\right|_{\alpha=0}$ for some canonical choice of the affine coordinate $\alpha$ which will be described below).

Proof. The first step is the convenient choice of the gauge (2): let $\alpha-X(\vartheta)=0$ be an (axially symmetric) maximal surface:

$$
\left.\mu^{\prime}\right|_{\alpha=X(\vartheta)}=0 \text {, }
$$

regular at least at one of the poles $\vartheta=0, \pi$. Using (4) and (5), we obtain an equation for $X$ :

$$
\left.\mu\right|_{\alpha=0}+\Psi_{2} X+(M-\Gamma+\Omega)\left(M^{+}+\Omega^{+}\right) X=0 .
$$

This can be written as

$$
\begin{gathered}
(M-\Gamma) M^{+} X+\left(\Omega M^{+}+\Omega^{+} M\right) X+\left(\Psi_{2}+M \Omega^{+}-\Gamma \Omega^{+}+\Omega \Omega^{+}\right) X \\
+\left.\mu\right|_{\alpha=0}=0 .
\end{gathered}
$$

I (32), (9) and the reality of $M(X$ is independent of $\alpha$ and $\varphi$ ) imply

$$
\begin{aligned}
M x+(2 \operatorname{Re} \Omega-\Gamma) x & =-\left.\mu\right|_{\alpha=0}, \\
x & =M X .
\end{aligned}
$$

Setting for $M, \Omega, \Gamma$ from (9) and (10) in (13), we solve easily for $x$ :

$$
x=\frac{C-\left.m \sqrt{2} \int_{0}^{\zeta}\left(1+\cos ^{2} z\right) \sin z \mu(z)\right|_{\alpha=0} d z}{\sin \zeta \sqrt{1+\cos ^{2} \zeta}}
$$

where $C$ is an arbitrary constant. $\left.\mu\right|_{\alpha=0}$ is regular at both poles, because $\alpha$ is a globally regular coordinate. Then, $x$ will be regular at $\zeta=0$, if $C=C_{0}=0$; at $\zeta=\pi$, if $C=C_{\pi}$, where

or

$$
C_{\pi}=\left.m \sqrt{2} \int_{0}^{\pi}\left(1+\cos ^{2} \zeta\right) \sin \zeta \mu(\zeta)\right|_{\alpha=0} d \zeta
$$

$$
\begin{aligned}
& x_{0}=-\left.\frac{m \sqrt{2}}{\sin \zeta \sqrt{1+\cos ^{2} \zeta}} \int_{0}^{\zeta}\left(1+\cos ^{2} z\right) \sin z \mu(z)\right|_{\alpha=0} d z, \\
& x_{\pi}=-\left.\frac{m \sqrt{2}}{\sin \zeta \sqrt{1+\cos ^{2} \zeta}} \int_{0}^{\zeta}\left(1+\cos ^{2} z\right) \sin z \mu(z)\right|_{\alpha=0} d z
\end{aligned}
$$


$\left[x_{0}\left(x_{\pi}\right)\right.$ is the solution of (13) which is regular at $\left.\zeta=0(\zeta=\pi)\right]$. Thus, there are two cases to distinguish:

(a) $\left(C_{\pi} \neq 0\right)$ : there is no maximal surface regular at both poles. For each value $\alpha$ of the affine coordinate there is just one maximal surface intersecting a given pole ray at $\alpha$ and being smooth there, but it does not reach the opposit pole ray at finite $\alpha$. One can use the remaining gauge freedom (3), (7) to normalize $C_{\pi}=\sqrt{2} \mathrm{~m}^{-2}$.

(b) $\left(C_{\pi}=0\right)$ : for each value $\alpha$ there is just one maximal Cauchy surface smooth at both poles.

The relation (15) shows that our gauge transformation is always possible; the system (8) is, then, equivalent to

$$
\begin{aligned}
\left(M^{+}+\Gamma^{+}+5 \Omega^{+}\right)(M-2 \Gamma+\Omega) \lambda^{\prime}+3 \Psi_{2} \lambda^{\prime} & =0, \\
\left.\Psi_{3}^{\prime}\right|_{\alpha=X} & =-(M-2 \Gamma+\Omega) \lambda^{\prime} .
\end{aligned}
$$

(16) is an ordinary, second order, linear, homogeneous differential equation with singular points at $\zeta=0, \pi$, for the complex function $\lambda^{\prime}$ of real variable $\zeta$, and (17) yields the value of $\left.\Psi_{3}^{\prime}\right|_{\alpha=x}$ correponding to a solution $\lambda^{\prime}$ of (16).

Lemma 15. All solutions $\lambda_{0}^{\prime}(\zeta)\left(\lambda_{\pi}^{\prime}(\zeta)\right)$ of Eq. (16) which are regular at the pole $\zeta=0(\zeta=\pi)$ are given by

$$
\left.\begin{array}{l}
\lambda_{0}^{\prime}=\bar{\lambda}_{0} \cdot\left(-\frac{i}{2 m^{3}}\right) \frac{(\cos \zeta-1)(\cos \zeta+2)}{(1-i \cos \zeta)^{2}(\cos \zeta+1)}, \\
\lambda_{\pi}^{\prime}=\bar{\lambda}_{\pi} \cdot\left(-\frac{i}{2 m^{3}}\right) \frac{(\cos \zeta+1)(\cos \zeta-2)}{(1-i \cos \zeta)^{2}(\cos \zeta-1)},
\end{array}\right\}
$$

where $\bar{\lambda}_{0}$ and $\bar{\lambda}_{\pi}$ are arbitrary complex constants. The values $\bar{\lambda}_{0}=\bar{\lambda}_{\pi}=1$ correspond to the extreme Kerr horizon $(a=m)$, if $\alpha$ is chosen, in each case

$$
\left.\begin{array}{l}
\alpha_{0}=\frac{u}{2 m^{2}}-\frac{1}{m} \lg (1+\cos \zeta), \\
\alpha_{\pi}=\frac{u}{2 m^{2}}-\frac{1}{m} \lg (1-\cos \zeta),
\end{array}\right\}
$$

where $u$ and $\zeta$ in the right hand sides of (19) are identical with the functions $u$ and $\vartheta$, respectively, from the four coordinates $u, r, \vartheta, \varphi$ used by Carter [6] to describe the Kerr-Newman family $\left(\alpha_{0, \pi}=\right.$ const are maximal surfaces regular at $\zeta=0, \pi$, respectively).

Proof of the Lemma 15. A straightforward but tedious calculation making use of the extreme Kerr horizon values as given, e.g. in [7] and employing (4), (14), and (15) yields (19) and (18) with $\bar{\lambda}_{0}=\bar{\lambda}_{\pi}=1$; at each pole $\zeta=0, \pi$, one of these functions $\lambda_{0}^{\prime}, \lambda_{\pi}^{\prime}$ is regular and one is singular. 
They are linearly independent, so any solution of (16) is a linear combination of them with complex coefficients. But, if both coefficients are non-zero, then the resulting solution would be singular at both poles, so the only possibility is (18), which proves the lemma.

The last step in the proof of the Theorem 7 initiates at the observation that the constants $\bar{\lambda}_{0}$ and $\bar{\lambda}_{\pi}$ cannot be completely arbitrary and independent of each other, if they both refer to the same horizon. In fact, $\lambda_{0}^{\prime}$ is computed in the frame $L, M^{\prime}$, where $M^{\prime}$ is tangential to the surface $\alpha-X_{0}(\vartheta)=0$ and analogously $\lambda_{\pi}^{\prime}$. Hence, according to (6) and (4)

$$
\lambda_{\pi}^{\prime}-\lambda_{0}^{\prime}=\left(M^{+}+\Gamma^{+}+\Omega^{+}\right)\left(M^{+}+\Omega^{+}\right)\left(X_{\pi}-X_{0}\right) .
$$

From (9), (10), and (15), we have

or

$$
\frac{d\left(X_{\pi}-X_{0}\right)}{d \zeta}=\frac{\sqrt{2} m C_{\pi}}{\sin \zeta},
$$

$$
X_{\pi}-X_{0}=\sqrt{2} m C_{\pi}\left(\bar{C}+\lg \sqrt{\frac{1-\cos \zeta}{1+\cos \zeta}}\right),
$$

where $\bar{C}$ is an arbitrary constant. Thus,

$$
\begin{aligned}
\lambda_{\pi}^{\prime}-\lambda_{0}^{\prime}= & \sqrt{2} m C_{\pi} \bar{C}\left(M^{+}+\Gamma^{+}+\Omega^{+}\right) \Omega^{+} \\
& +\sqrt{2} m C_{\pi}\left(M^{+}+\Gamma^{+}+\Omega^{+}\right)\left(M^{+}+\Omega^{+}\right) \lg \sqrt{\frac{1-\cos \zeta}{1+\cos \zeta}} .
\end{aligned}
$$

The first term on the right hand side is zero by virtue of I (31) and we obtain in the two cases (a) and (b):

(a) $\lambda_{\pi}^{\prime}-\lambda_{0}^{\prime}=\left(M^{+}+\Gamma^{+}+\Omega^{+}\right)\left(M^{+}+\Omega^{+}\right)$

$$
\cdot\left[\frac{1}{m} \lg (1-\cos \zeta)-\frac{1}{m} \lg (1+\cos \zeta)\right] ;
$$

from (19) and (20) it follows that $\lambda_{\pi}^{\prime}-\lambda_{0}^{\prime}$ is, in this case, exactly equal to the difference between the corresponding extreme Kerr values, e.g. necessarily $\bar{\lambda}_{0}=\bar{\lambda}_{\pi}=1$. But then, the horizon is clearly the Kerr one with $a=m$.

(b) $\lambda_{\pi}^{\prime}-\lambda_{0}^{\prime}=0$;

from (18) it follows that this is only possible, if $\bar{\lambda}_{0}=\bar{\lambda}_{\pi}=0$. But then, the maximal surface $\alpha=X(\vartheta)$ satisfies $\lambda^{\prime}=0$ in addition to $\left.\mu^{\prime}\right|_{\alpha=X(\vartheta)}=0$, and must, therefore, be a totally autoparallel Cauchy surface, which, together with (17) and Lemma 6 of I, proves the Theorem 7.

There are a few comments on the Theorem 7. The freedom in $\Phi_{2}$ and $\Psi_{4}$ probably means that the space-times containing any AT-horizon 
with $\Phi_{1}=0$ are very numerous, because it seems likely that there is at least one axisymmetric stationary space-time for any allowed value of $\Phi_{2}, \Psi_{4}$. In addition to that, complete characteristic initial data must be given along two intersecting null hypersurfaces [8] in order to determine the solution uniquely. The AT-horizons consist of only one null hypersurface unlike the bifurcate AC-horizons. Thus, one ought to choose another null hypersurface intersecting the AT-horizon and specify the rest of initial data along it. On the other hand, if the resulting space-time should be axisymmetric and stationary, one cannot prescribe the data along the second null hypersurface completely arbitrarily. The question is how much freedom can remain.

Concerning (b) of the theorem, it is of interest to construct at least one space-time containing a (b)-horizon. If we require that the full ACT-symmetry of the horizon be induced by a space-time symmetry, then there is no remaining freedom in $\Phi_{2}$ and $\Psi_{4}$

$$
\Phi_{2}=0, \quad \Psi_{4}=6\left(\Omega^{+}\right)^{2} \Psi_{2} \alpha^{2} .
$$

This space-time (if it exists) would be pure vacuum. The horizon would be bifurcate at any surface $\alpha=$ const and so would all the branch horizons, because they must have the same structure as the original one (see II), i.e. any point $p$ of the corresponding space-time lies on two perfect horizons. Thus, this space-time cannot be asymptotically flat. On the other hand, it is conceivable that an axisymmetric stationary shell of reasonable matter surrounding the horizon can separate such an inner metric from a more sensible outer one. If this proves to be impossible, and if even all horizons of the (b)-family turn out to have only asymptotically non-flat surroundings, then we would arrive at some sort of exclusivity of the extreme Kerr horizon.

\section{Surface Area, Angular Momentum, and Charges of Axisymmetric Horizons}

In II it was shown that the AC-horizons determine their axisymmetric stationary surrounding space-times uniquely, so that the characteristic parameters of the corresponding black hole such as the total mass $M$, total surface area $A$, total angular momentum $J$, total electric charge $Q$ (and magnetic $H$ ), can be computed, at least in principle. Concerning the AT-horizons, some Kerr-Newman-like parameters $m, a, e, h$ were formally defined, but their possible relation to $M, A, J, Q, H$ was not clarified. It is the purpose of the present section to calculate the parameters $A, J, Q, H$ from the horizon structure of an axisymmetric perfect horizon $\mathscr{M}$. 
First, we choose a convenient reference frame on $\mathscr{M}$. The coordinates $\alpha, \vartheta, \varphi$ always exist such that the line element of the horizon is

$$
d s^{2}=-R^{2}\left(d \vartheta^{2}+A^{2}(\vartheta) d \varphi^{2}\right),
$$

$\partial / \partial \varphi$ being the generator of the axial horizon symmetry and $\alpha$ an affine coordinate satisfying,

$$
\left[\frac{\partial}{\partial \alpha}, \frac{1}{\partial \varphi}\right]=0,
$$

that is to say, the vector field

$$
L=\frac{\partial}{\partial \alpha}
$$

is axially symmetric and $\nabla_{L} L=0$. Finally, if

$$
M=\frac{1}{\sqrt{2}} \frac{1}{R}\left(\frac{\partial}{\partial \vartheta}+\frac{i}{A(\vartheta)} \frac{\partial}{\partial \varphi}\right)-\alpha \cdot \Omega(\vartheta) \frac{\partial}{\partial \alpha},
$$

then $[M, L]=\Omega L$, and we obtain $\nabla_{L} M=0$ defining $\Omega$ by

$$
\frac{1}{\sqrt{2}} \frac{1}{R}\left(\nabla_{\frac{\partial}{\partial \vartheta}}+\frac{i}{A(\vartheta)} \nabla_{\frac{\partial}{\partial \varphi}}\right) L=\Omega L .
$$

Note that

where

$$
\nabla_{\frac{\partial}{\partial \varphi}} L=-\Omega_{\varphi} L,
$$

$$
\Omega_{\varphi}=-\sqrt{2} R A(-i \vartheta m \Omega)
$$

is the $\varphi$-component of the covariant vector $\Omega_{A}$ [see I (21)] (which is identical with the invariant $B(\vartheta)$ introduced in II). Let $\mathscr{M}$ be embedded in an axisymmetric space-time $\overline{\mathscr{M}}$,

$$
\theta: \mathscr{M} \rightarrow \overline{\mathscr{M}}
$$

such that

$$
\theta_{*}\left(\frac{\partial}{\partial \varphi}\right)=\left.\tilde{K}\right|_{\mathscr{M}},
$$

where $\tilde{K}$ is the Killing vector field of the axisymmetric group normalized so that the group parameter spans the interval $(0,2 \pi)$; choose an axisymmetric space-like hypersurface $\bar{S}$ in $\overline{\mathscr{M}}$ intersecting the horizon $\mathscr{M}$ at a surface $\partial S$ with $\alpha=0$ at $\partial S$. There is a unique pseudo-orthonormal tetrad $\bar{L}, \bar{N}, \bar{M}, \bar{M}^{+}$along $\mathscr{M}$ in $\bar{M}$ such that $\bar{N}$ is future oriented and

$$
\theta_{*}(L)=\bar{L}, \quad \theta_{*}(M)=\bar{M} .
$$


The total surface area $A$ of $\partial S$ is clearly given by

$$
A=\int_{0}^{\pi} d \vartheta \int_{0}^{2 \pi} d \varphi \sqrt{g},
$$

or, using (21)

$$
A=2 \pi R^{2} \int_{0}^{\pi} A(\vartheta) d \vartheta
$$

Introduce the function $p(\vartheta)$ :

$$
p(\vartheta)=2 \pi R^{2} \int_{0}^{\vartheta} A(z) d z
$$

so that $\sqrt{g}=p^{\prime} / 2 \pi, A=\dot{p}(\pi)$.

The total angular momentum $J$, as defined by Bardeen, Carter and Hawking [3], is given by

$$
J=-\frac{1}{8 \pi} \int \tilde{K}^{a ; b} d \Sigma_{a b}
$$

where one integrates along $\partial S$, the semicolon denoting the covariant derivative with respect to the affine connection $\bar{\nabla}$ of $\overline{\mathscr{M}}$ and

$$
d \Sigma_{a b}=\bar{L}_{[a} \bar{N}_{b]} \sqrt{g} d \vartheta d \varphi .
$$

Because $\tilde{K}^{a ; b}$ is antisymmetric in $a$ and $b$, we obtain

$$
J=\frac{1}{8 \pi} \int_{\partial S} \tilde{K}_{a ; b} \bar{N}^{a} \bar{L}^{b} \sqrt{g} d \vartheta d \varphi
$$

On the other hand, developing $\bar{\nabla}_{\bar{L}} \tilde{K}$ in the pseudo-orthonormal tetrad $\bar{L}, \bar{N}, \bar{M}, \bar{M}^{+}$:

$$
\bar{\nabla}_{\bar{L}} \tilde{K}=\left(\tilde{K}_{a ; b} \bar{N}^{a} \bar{L}^{b}\right) \bar{L}-\left(\tilde{K}_{a ; b} \bar{M}^{a} \bar{L}^{b}\right)^{+} \bar{M}-\left(\tilde{K}_{a ; b} \bar{M}^{a} \bar{L}^{b}\right) \bar{M}^{+},
$$

applying $\theta_{*}^{-1}$ to both sides of this relation and using I (2), (27), and (28), we obtain

$$
\nabla_{L} \frac{\partial}{\partial \varphi}=\left(\tilde{K}_{a ; b} \bar{N}^{a} \bar{L}^{b}\right) L-\left(\tilde{K}_{a ; b} \bar{M}^{a} \bar{L}^{b}\right)^{+} M-\left(\tilde{K}_{a ; b} \bar{M}^{a} \bar{L}^{b}\right) M^{+} .
$$

But (22), (23), and (25) imply

$$
\nabla_{L} \frac{\partial}{\partial \varphi}=-\Omega_{\varphi} L
$$


Comparing (32) and (33) yields, together with (31)

$$
J=-\frac{1}{8 \pi} \int_{\partial S} \Omega_{\varphi} \sqrt{g} d \vartheta d \varphi .
$$

Using the relation (30) and I (23) and integrating by parts, we express the angular momentum in terms of the invariant $\Psi_{2}$ :

$$
J=-\frac{1}{8 \pi^{2}} \int_{0}^{\pi}\left(-i \operatorname{Im} \Psi_{2}\right) p p^{\prime} d \vartheta
$$

because $\Omega_{\varphi}(0)=\Omega_{\varphi}(\pi)=0$.

The geometric meaning of $\Omega_{\varphi}$ is as follows: parallel transport of any vector $L^{\prime}$ tangential to the rays along the closed group orbit $\alpha=$ const, $\vartheta=$ const from $\varphi=0$ to $\varphi=2 \pi$ results in $\exp \left(-2 \pi \Omega_{\varphi}\right) \cdot L^{\prime}$. Thus, $\Omega_{\varphi}$ is an invariant, and $J$ is independent of $S$ and $\partial S$.

Charges $Q$ and $H$. The meaning of the invariant $\Phi_{1}$ is, according to [9]

$$
\Phi_{1}=\frac{1}{2}\left[F_{a b} \bar{L}^{a} \bar{N}^{b}+F_{a b}\left(\bar{M}^{a}\right)^{+} \bar{M}^{b}\right] .
$$

Let us introduce the real orthonormal tetrad $e_{a}^{i}$ with radial vector $e_{1}^{i}$ oriented out of the sphere $\partial S$ :

$$
\bar{L}^{i}=\frac{1}{\sqrt{2}}\left(e_{0}^{i}+e_{1}^{i}\right), \quad \bar{N}^{i}=\frac{1}{\sqrt{2}}\left(e_{0}^{i}-e_{1}^{i}\right), \quad \bar{M}^{i}=\frac{1}{\sqrt{2}}\left(e_{2}^{i}-i e_{3}^{i}\right) .
$$

The affine coordinate $\alpha$ can always be further specialized so that $e_{0}^{i}$ is orthogonal to $\bar{S}$ : because $\bar{S}$ and $\alpha$ are axially symmetric the corresponding transformation (if necessary) of $\alpha$ will not disturb the relation (22). Then

$$
\Phi_{1}=\frac{1}{2}\left(E^{1}-i H^{1}\right),
$$

Where $E^{1}$ and $H^{1}$ are the outer $\partial S$-normal components of $E^{i}$ and $H^{i}$ as measured by an observer moving along $e_{0}^{i}$. Then, the theorem of Gauss implies

$$
Q-i H=\frac{1}{2 \pi} \int_{\partial S} \Phi_{1} \sqrt{g} d \vartheta d \varphi=R^{2} \int_{0}^{\pi} \Phi_{1} A d \vartheta
$$

The magnitudes of $E^{1}$ and $H^{1}$ are, in fact, independent of any observer and hypersurface $\bar{S}$, because $\Phi_{1}$ is an invariant (see II).

The simple formulae (29), (34), and (36) determine four of the five black hole parameters from the corresponding horizon structure. In case the horizon has some longitudinal symmetry in addition to the axial one, then there should be a well-definable total mass $M$ of the corresponding black hole (if there is only one stationary axisymmetric space-time for the horizon). But $M$ is difficult to obtain, because it is defined by a 
Killing vector field $K$ which is time-like and unit at the infinity of $\bar{M}$ [3], and so we are as yet not able to calculate $M$ directly from the local horizon data: we do not know the two factors in $K=a L+b \frac{\partial}{\partial \varphi}$.

Concerning the AT-horizons, the formulae (34) and (36) imply that our Kerr-Newman-like parameters $m, a, e, h$ as defined in II have, at least partially, not only a formal meaning. Indeed,

$$
\begin{gathered}
J=-m a+\left(e^{2}+h^{2}\right) \frac{\left(r_{+}^{2}+a^{2}\right)^{2} \operatorname{arctg} \frac{a}{r_{+}}-a r_{+}\left(r_{+}^{2}-a^{2}\right)^{1}}{4 a^{2} r_{+}^{2}}, \\
Q-i H=e-i h,
\end{gathered}
$$

where

$$
r_{+}=m+\sqrt{m^{2}-a^{2}-e^{2}-h^{2}} .
$$

\section{Schwarzschild Black Hole in Weak External Field}

In II the question remained open what shape of horizon corresponds to a given matter and charge content of the outer space-time, and what is the field around the horizon.

In the present section the last question is answered for a very simplified model. Its main features are

1) exact stationarity and axisymmetry,

2) the matter and charge produce only a small perturbation of the background geometry,

3) the background geometry is Schwarzschild,

4) the matter and charge are concentrated on a spherical surface layer at $r=R_{0}\left(2 m<R_{0}<\infty\right)$.

Such a shell is mathematically described as a jump in the $r$-derivative of the metric and vector potential (see, e.g. [11]). The matter of the shell must satisfy three groups of conditions:

1) the validity of Einstein-Maxwell equations outside the shell imply the equations of motion of matter and conservation of charge [11],

2) The distribution and motion of matter and charge must be stationary and axisymmetric,

3) the stress-energy tensor $\tau^{a b}$ of matter must have one time- or light-like eigenvector with positive eigenvalue and the dominant energy condition should not be violated.

\footnotetext{
${ }^{1}$ Note that for the Kerr-Newman black hole with non-zero charges, $J \neq-m a$; the minus sign associated with $m a$ appears, because the hole rotates backwards in the Carter coordinates [6] we are using (see also [10]).
} 
Several (unpublished) models have been constructed showing that these conditions are compatible. I emphasize: no attempt is made to claim that such a (possibly) differentially rotating, soap bubble with negative surface tension can really be constructed from conceivable material or even exist in astrophysical systems. It is merely believed that some qualitative features of the model could have some general validity.

A part of the well-known results $[12,13]$ on perturbations of Schwarzschild background can be summarized as follows:

The stationary, axisymmetric $\pi, l$-multipole perturbation of a spin $S$ field ${ }^{2}$ consists, for any $l$ and $\pi$ (except for $l=0$ ) of two linearly independent, unique, modes, $\Phi_{S l \pi}^{1}, \Phi_{S l \pi}^{2}$ such that, say

1) their radial parts equal +1 at $r=R_{0}$,

2) $\Phi_{S l \pi}^{1}$ vanishes at $\infty$ as $r^{-l}$,

3) $\Phi_{S l \pi}^{2}$ is regular at the horizon, but is non-zero (or diverging) at $\infty$. $\Phi_{S l \pi}^{1}$ is regular at the horizon only if $l<S$.

Hence, the most general regular solutions of a given $S, l, \pi$ in the two regions of the model are

I. $\left(R_{0}<r<\infty\right): A^{S l \pi} \Phi_{S l \pi}^{1}$,

II. $\left(2 m<r<R_{0}\right): B^{S l \pi} \Phi_{S l \pi}^{1}+C^{S l \pi} \Phi_{S l \pi}^{2} \quad$ if $\quad l<S$,

$$
C^{S l \pi} \Phi_{S l \pi}^{2} \quad \text { if } l \geqq S,
$$

where $A^{S l \pi}, B^{S l \pi}$, and $C^{S l \pi}$ are arbitrary constants. The continuity at $r=R_{0}$ requires (see the Condition 1))

$$
\begin{array}{llrl}
A^{S l \pi}=B^{S l \pi}+C^{S l \pi}, & & \text { if } & l<S, \\
A^{S l \pi}=C^{S l \pi}, & \text { if } & l \geqq S .
\end{array}
$$

Thus, there are two distinct types of continuous modes:

Hole modes:

$$
B^{S l \pi} \Phi_{S l \pi}^{1} \text { in I. }+ \text { II., for } l<S,
$$

Matter modes:

$$
C^{S l \pi} \Phi_{S l \pi}^{1} \quad \text { in I., } \quad C^{S l \pi} \Phi_{S l \pi}^{2} \quad \text { in II., for any } l .
$$

The hole modes are analytic throughout the spacetime and couple to a small amount of mass $(S=2, l=0, \pi=0)$, angular momentum $(S=2$, $l=1, \pi=1)$, and charge $(S=1, l=0, \pi=0)$ added to the black hole. The

${ }^{2} l$ is the total angular momentum, $(-1)^{\pi+l}$ is the parity and $S$ is 2 for gravitational, 1 for electromagnetic perturbation mode. 
matter modes couple to the $S, l, \pi$-part of the shell stress-energy tensor $(S=2)$ and electric current $(S=1)$ showing the corresponding jump at $r=R_{0}$. The decomposition of the shell stress-energy tensor into spherical harmonics is unique. The coefficients of the decomposition determine the corresponding coefficients $C^{S l \pi}$ without ambiguity. Thus, in this special model, the stationary space-time, or, with other words, the equilibrium configuration of the system hole - matter shell, is fixed, if we specify

1) total mass, angular momentum and charge of the black hole,

2) stress-energy tensor of matter (density + angular velocity, stress being determined by the equations of motion),

3) density and angular velocity of charge.

There is, therefore, a clear sense in which one can say that the equilibrium degrees of freedom of the black hole are given by the three parameters 1). If we assume the same not only for our special model, but for all stationary situations, we obtain a sort of generalized no hair conjecture.

But the field around the hole is not uniquely determined by the three parameters 1). One must consider the modes $\Phi_{S l \pi}^{2}$, because they describe the part of the perturbation between the shell and the horizon that produce a deformation of the horizon from its standard Kerr-Newman shape. Let us describe an example with pure magnetic perturbation (total charge of the shell as well as that of the hole is zero). The example could even have some direct physical value: As it is currently believed (see, e.g. [14]), the more collapsed an astrophysical system is, the more significant is the role played by rotation and the magnetic field. For vacuum black holes (which could be the most collapsed astrophysical objects at all), the validity of the rule cannot be extended, because the magnetic field of the original star must be completely radiated away [13] (if the hole has no net electric charge). On the other hand, if the endproduct of a collapse is a hole surrounded by matter, no proof for "radiating away" exists, and the conjecture that some of the original enormous magnetic field remains captured in the matter, even if the resulting hole is electrically neutral, need not seem a priori wrong.

Price's equation [13]

$$
\left(r^{2} \Phi_{0}\right)_{, r_{r}^{*} *}-\left(1-\frac{2 m}{r}\right) \frac{l(l+1)}{r^{2}}\left(r^{2} \Phi_{0}\right)=0
$$

for the stationary axisymmetric $l$-partial wave of $\Phi_{0}$-component of Maxwell spinor has solutions

$$
\Phi_{0}=r^{-2} Q_{l}\left(\frac{r}{2 m}\right)
$$


where $Q_{0}(x)=1$, and $Q_{l}(x)$, if $l \geqq 1$, are polynomials with lowest power 2 , of degree $l+1$, whose coefficients are given by the recurrent formula

$$
a_{n+1}=a_{n} \frac{(n-1) n-l(l+1)}{(n-1)(n+1)} .
$$

If $l \geqq 1$, each of these $\Phi_{0}$ 's is regular at the horizon and non-zero at $\infty$. Hence, they represent our matter modes for $l \geqq 1$. The magnetic dipole contribution is

$$
\Phi_{0}=-\frac{i}{2} B_{0} \cos \vartheta
$$

where $B_{0}$ is some real constant. The corresponding magnetic field components in the orthonormal tetrad associated with a freely-falling observer, who starts from rest in spacial infinity (for exact definition, see [13]) are,

$$
\tilde{B}^{r}=B_{0} \cos \vartheta, \quad \tilde{B}^{\vartheta}=-B_{0} \sin \vartheta, \quad \tilde{B}^{\varphi}=0 .
$$

We have a "homogeneous" magnetic field, which is exactly as strong at the horizon as at the shell.

In face of this naturally looking result, one is tempted to conjecture that a charged ring or disk can produce a sort of solenoidal field which would be even stronger at the horizon than at the source.

There is still a point to be settled. A theorem was proved [15] that black holes behave like exact conductors, i.e. the electromagnetic field at the horizon must be purely radial. But this is only true for the fields in a limiting stationary frame, which is singular at the horizon, because its radial and time-like unit vectors collapse into the null direction of the horizon rays. Hence, there is no contradiction to our field (37), which is written in a regular time-like frame.

\section{References}

1. Metz, W.D.: Science 179, 1113 (1973)

2. Thorne, K.S.: Lecture at the Summer School, Les Houches, 1972

3. Bardeen,J.M., Carter,B., Hawking,S.W.: Commun. math. Phys. 31, 161 (1973)

4. Israel, W.: Commun. math. Phys. 8, 245 (1968)

Carter, B.: Phys. Rev. Letters 26, 331 (1971)

5. Hájiček,P.: Lettere Nuovo Cimento 6, 157 (1973); Commun. math. Phys. 34, 37 (Part I); 34, 53 (Part II)

6. Carter, B.: Phys. Rev. 174, 1559 (1968)

7. Teukolsky,S.A.: Gravitational perturbations of a rotating black hole. Orange Aid Preprints 269, December 1971

8. Sachs, R. K.: J. Math. Phys. 3, 908 (1962) 
9. Newman,E.T., Penrose, R.: J. Math. Phys. 3, 566 (1962)

10. Stewart,J., Walker,M.: Black Holes: the outside story. Springer Tracts in Modern Physics 69, 69 (1973)

11. Israel, W.: Nuovo Cimento 45B, 1 (1966)

Kuchař, K.: Czech. J. Phys. B 18, 435 (1968)

12. Regge,T., Wheeler, J.A.: Phys. Rev. 108, 1063 (1957)

Vishveshwara, C.V.: Phys. Rev. D 1, 2870 (1970)

13. Price, R.: Phys. Rev. D 5, 2439 (1972)

14. Pacini,F., Rees, M.J.: Sci. Am., p. 98, Feb. 1973

15. Carter, B.: Preprint, Les Houches, 1972

Communicated by J. Ehlers

P. Hájiček

Institute for Theoretical Physics

University of Berne

Sidlerstraße 5

CH-3000 Berne, Switzerland 